\title{
Survey on Air Levitation Conveyors with possible scalability properties
}

\author{
Li Zhu, Didier El Baz \\ CNRS - LAAS, 7 av. du col. Roche F-31400 Toulouse \\ Université de Toulouse, LAAS, F-31400 Toulouse, \\ France \\ lzhu@laas.fr, elbaz@laas.fr
}

\author{
Huangsheng Ning \\ University of Science \& Technology Beijing \\ 30th Road, Haidian District, Beijing, China \\ ninghuansheng@ustb.edu.cn
}

\begin{abstract}
Re-configurability and scalability are important properties of smart non-contact conveyors. The paper studies smart conveyors. It concentrates on levitation methods; it makes a comprehensive survey on levitation technologies that are currently most active. A conclusion is made that air levitation is well suited to smart conveyors. Furthermore, an overview of the movement mechanisms on air levitation platforms is presented; the various advantages of these technologies are reviewed here and associated scalability properties are detailed. This provides a reference for selecting a reliable movement mechanism for future smart conveyors.
\end{abstract}

Keywords-scalability; smart conveyor; air levitation;
movement mechanism

\section{INTRODUCTION}

So far, conveyors have been designed to solve one problem at a time. Usually they lack reactivity to environment changes and failures that occur at small scales [1]. Thus, re-configurability and scalability will be important properties of future smart conveyors.

In industry, it's often necessary to manipulate and transport materials with thin and fragile surface textures, such as silicon wafers, LCD-displays and paper coated with wet ink. These materials are easily broken or damaged during transport and manipulation. Every physical contact with the product brings risk of breakage or contamination. A non-contact handling or conveying approach is highly desirable in these situations [2]. Levitation and controlled motion of matter in air have a wealth of potential applications ranging from materials processing to biochemistry and pharmaceuticals. There are many advantages of non-contact conveying approach, which can be summarized as follows [3]: firstly, the surface forces can be completely neglected, which enables high resolution and accuracy motion devices by avoiding the stick-slip effects [4]. Secondly, since high local contact pressure by direct mechanical contact is avoided, handling of tricky (fragile, freshly painted) components and non-rigid products is feasible $[5,6]$. Xerox Palo Alto uses this technology to transport paper [2]. Thirdly, contamination from the input to the output of the production line can be totally avoided. This can be important in food or drug handling [6] or in presence of lubricant [7] and in material science [8].

In this paper we present a survey on levitation techniques which could be used in smart conveyors and their possible scalability properties. The structure of this paper is as follows: section 2 deals with the numerous physical principles suitable for non-contact levitation. A comparison shows the air levitation is more suitable to smart conveyors. Different moving mechanisms of air levitation techniques and their scalability are discussed in section 3. The last section of this work presents some conclusions and perspectives.

\section{DIFFERENT TECHNOLOGIES OF NON-CONTACT LEVITATION}

To achieve non-contact conveying, two conditions are needed, i.e. levitation and moving the levitated objects. Currently the most active levitation technologies are Electromagnetic Levitation, Electrostatic Levitation, Ultrasonic Levitation and Air Levitation.

\section{A. Electromagnetic Levitation}

Electromagnetic levitation technology is one of the fastest growing areas of modern industrial technology. Its main principle is to use permanent magnets, electromagnets or superconducting magnets forming a magnetic field to provide driving force. In 1937, Kenper applied for the first maglev technology patents; he thought controllable electromagnet magnetic technology must be used to adjust the magnetic force constantly to achieve stable levitation, which became the guidelines of Electromagnetic Levitation.

From the beginning of 1990s, with the rapid development of semiconductor industry, the electromagnetic levitation technology began to seep into the non-contact transmission in the field of semiconductor materials. P.R. Southworth [9] and D. Belnap [10] both developed electromagnetic levitation transport systems for contactless transmission of semiconductor materials. K. H. Park [11, 12] developed a new type of electromagnetic levitation silicon wafer transmission system to solve the pollution problems in traditional contact transport.

However, electromagnetic levitation is only applicable to the conductor or semiconductor materials with high conductivity. Compared with it, electrostatic levitation has a wider application field.

\section{B. Electrostatic Levitation}

Objects in a static electrostatic field effect by the Coulomb force; when it equals to gravity, objects can levitate. So this technology is suitable for materials which can keep enough static charges on its surface, such as metal, semiconductor and insulator etc. In 1998, Higuchi [13, 14] achieved to make glass and other insulating materials suspended about $0.3 \mathrm{~mm}$. In 2007, Higuchi and Jeon [15] developed a non-contact transmission device to levitate a 
glass plate; it provides a lateral thrust to transfer the glass plate at the speed of $25.6 \mathrm{~mm} / \mathrm{s}$.

\section{Ultrasonic Levitation}

Ultrasonic Levitation phenomenon was first found by Kundt in 1886. When ultrasonic forms a standing wave it can hold objects. In 1988, Ueha builds an ultrasonic noncontact transmission system to transport silicon wafer [16]. In 2002, Xie's work shows single-axis acoustic levitation of the heaviest soil (iridium, $\rho=22.6 \mathrm{~g} / \mathrm{cm} 3$ ) and liquid (mercury, $\rho=13.6 \mathrm{~g} / \mathrm{cm} 3$ ) on the earth is achieved by greatly enhancing both the levitation force and stability through optimizing the geometric parameters of the self-developed single-axis acoustic levitator. This indicates that all the solids and liquids can be acoustically levitated on the earth in principle [17]. In 2014, Ochiai demonstrated an extended acoustic manipulation by which millimeter-sized particles can be levitated and moved three-dimensionally by localized ultrasonic standing waves generated by ultrasonic phased arrays [18]. Compared with electromagnetic levitation and electrostatic levitation, ultrasonic levitation is not restricted by the conductivity of materials, in this respect, it has a wider area; but it is not suitable for large objects, and its equipment construction cost is high, maintenance is also more difficult.

\section{Air Levitation}

Air levitation is based on the principles of fluid dynamics, it makes use of air to produce a pressure differential between the upper and lower surface of the object in order to balance its gravity. Researchers have experimented with a variety of air-jet techniques to design contactless manipulators. Depending on the principle, it divided into two different approaches: Bernoulli adsorption and Air cushion.

In Bernoulli levitation, the sample is held below the endeffector of the manipulator which consists of a cup-shaped air nozzle. Since the middle of last century, people have carried out the study of the radial flow between two parallel flat plates, and the designed some simple suction floating machine. This is earlier application of the Bernoulli levitation in the industry. Now it has been used in practical applications to "pick-and-place" wafers [19] and flat soft foodstuffs [20]. Air cushion technology was originally developed as a pair of hydrostatic bearing, the supporting function on the work piece in precision machining, the basic principle is to form a layer of air pressure film bearing load so as to achieve non-contact. This is also the principle of popular air-hockey tables [21].

The air levitation has several advantages such as clean, magnetic free and generating little heat, simple in design, easy to use and maintenance, and has less demanding on the environment (such as antimagnetic, explosion, fire etc.) and therefore comes to dominate in then on-contact conveying field.

\section{DIFFERENT MOVING MECHANISMS OF AIR LEVITATION}

Researchers have developed many kinds of air levitation devices, the basic principles of them are the same; objects are levitated by air cushion. But the way of moving the levitated objects are totally different. We summarize them into three categories: inclined air jet, aerodynamic-traction and other non-flow integrated approaches.

\section{A. Moving objects by inclined air jet}

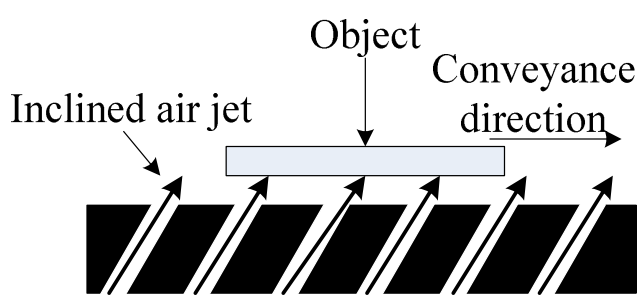

Figure 1.Basic structure of inclined air-jets principle.

Fig. 1 shows the basic structure of the moving mechanism based on inclined air-flow provided by tilted apertures. Several micro-fabrication techniques (dry and wet etching) can be used to fabricate inclined holes, e.g. nozzles, such as laser drilling [22], EDM (electrical discharge machining) [23] and FIB (focused ion beam)[24, 25]. It also can be seen, that apertures are fixed and objects can move only in the direction which was established at first. If objects need to change direction, then the apertures have to be redesigned, and this step can't be achieved during the process. This is certainly not a desirable way for scalable and reconfigurable systems. Therefore, researchers have found many ways to extend moving mechanisms.

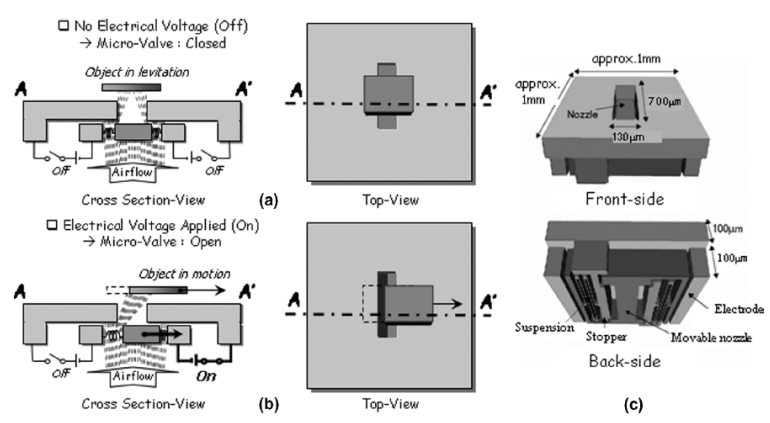

Figure 2. Principles of the pneumatic actuator according to the on/off voltage supply, given by the cross-section and top view of the device with the object onto it. (a) No electrical voltage. (b) Electrical voltage applied.

Fukuta's team proposes a distributed-air-jet MEMSbased system to manipulate small parts with high velocities and without any friction problems [26]. This system is composed of a $35 * 35 \mathrm{~mm}^{2}$ area and 560 microactuators. The experiments show the velocity of this system is between $0.5 \mathrm{~mm} / \mathrm{s}$ and $4.5 \mathrm{~mm} / \mathrm{s}$. And the maximum force is $0.37 \mu \mathrm{N}$ with a pressure of $13.2 \mathrm{kPa}$.

Fig. 2 displays the cross-section and top view of the pneumatic microactuator which is proposed by Mita [27]. The pneumatic microactuator consists of two layers: a bottom layer composed of a movable valve actuated by an electrostatic microactuator, and a top layer with a hole in it. Once an electrical voltage is applied between electrode and 
suspension beams, an electrostatic force is generated to open the movable valve, then the air-flow comes from the backside through the hole in the pneumatic microactuator. When there is no electrical voltage supply, a vertical airflow is generated to levitate the object, as shown in Fig. 2(a). In order to move the levitated object, such as move it to the right. An electrical voltage is applied in the right part of the electrostatic microactuator, as a result, the hole on the right side will close, airflow only comes out from the hole on the left side, the object will be blowed to the right, as shown in Fig. 2(b). Fig. 2(c) presents a three-dimensional prototype of the pneumatic microactuator, it consists of two silicon substrates: front-side and back-side. A hole is designed in the front-side and the back-side includes the structure of the electrostatic microactuator-based movable valve.

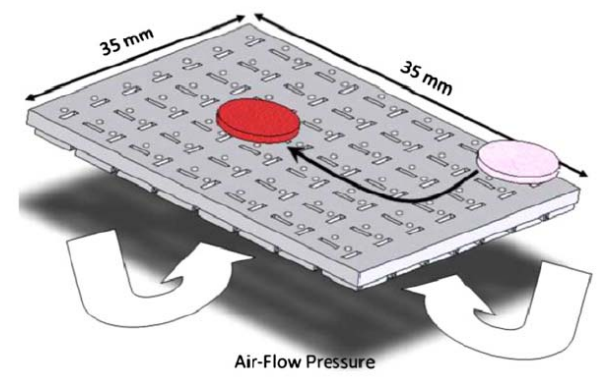

Figure 3. General overview of the surface

Based on this system, an original algorithm for distributed part differentiation was designed and several distributed synchronous and asynchronous discrete state acquisition algorithms and some stopping criteria were proposed [28]. Fig. 3 shows a general overview of the surface, from which we can see, scalability of this approach can be ensured by adding more systems in order to design a larger test bed.

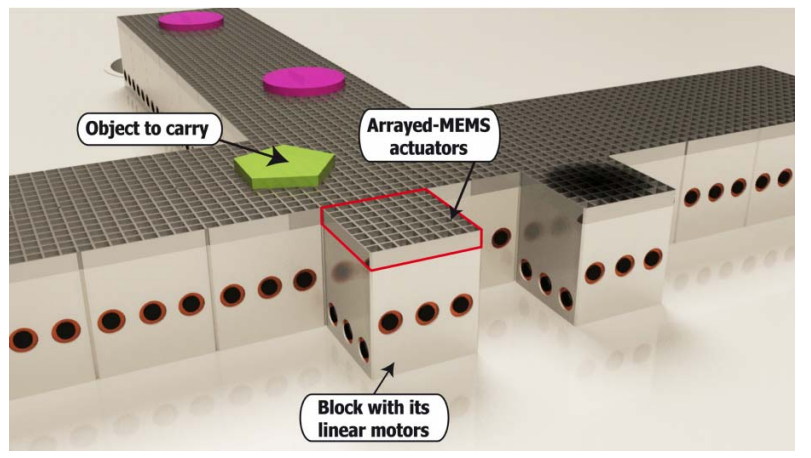

Figure 4. Smart conveyor

The Smart Blocks project [29, 30] is an extension of smart surface project. This project was funded by the French National Agency for Research (ANR). It has federated four French research laboratories and one Japanese laboratory. Its goal was to create a self-reconfigurable modular conveyor based on a contact-free technology. This conveyor is composed of centimeter-size blocks $(1-3 \mathrm{~cm})$, called smart blocks, which are linked together to form the conveying surface (Fig. 4). Re-configurability and scalability are highly considered in this project. Basically, these features come from the distributed nature of the smart conveyor. One can easily add blocks in order to extend the conveyor capabilities. Fig. 5 displays basic block motion that can be implemented with block prototypes via electro-permanent (EP) magnets.
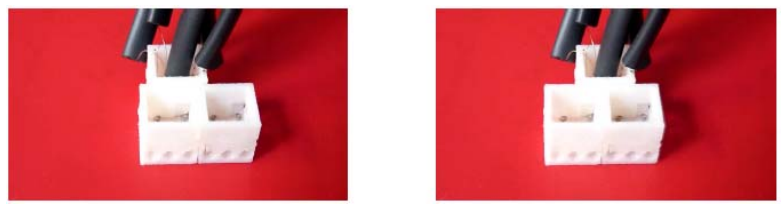

Figure 5. Photo of block prototypes and possible motion

Konishi and Fujita designed a fluidic micro actuator which has two on-off nozzles and fabricated an array of these micro actuators on an SOI (Silicon On Insulator) substrate for transporting tiny objects. The dimension of each actuator is about $100 \mu \mathrm{m} * 200 \mu \mathrm{m}$. Each micro actuator can control the direction of air flow by electrostatically closing one of nozzles and can carry a flat object on the flow to the desired direction.

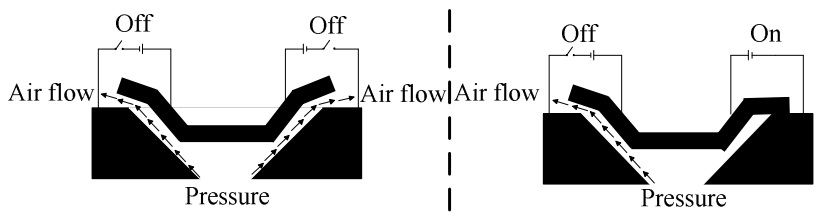

Figure 5. Mechanism of the motion of an actuator (cross-section . (a) Normalsection (b) When voltage is applied to one electrode.

Fig. 5 shows the principle of this actuator. The nozzle is normally open. When pressure is supplied from backside, air flows at an angle aslant from nozzles. Electrostatic force between the electrodes and the substrate is used to close nozzles. When the voltage is applied between the electrode and the substrate, the nozzle is squeezed down and closed by electrostatic force. When one nozzle is closed, the air can only flow through the other nozzle. As a result, the direction of the air flow can be controlled. Furthermore, if using a pair of actuators arranged orthogonally each other, then it can convey objects in four directions [31].

\section{B. Moving objects by aerodynamic-traction}

With the increasing complexity of industrial transport, advanced tasks such as high precision and manipulating many objects along arbitrary paths are required. A more complicated moving mechanism is invented: aerodynamictraction, it's a manipulation of the pressure field to move objects.

$\mathrm{Ku}$ has presented a system, called ASD (active surface device). It is a new type of automation concept to handle multiple objects with high precision. The concept consists of using a massively parallel micro actuator array to generate a pressure field on a planar surface. The objects can be translated, rotated, and flipped by controlling the pressure field surrounding the objects. It is worth 
mentioning that, the general architecture of this system is modular, which allows the system to be expanded.

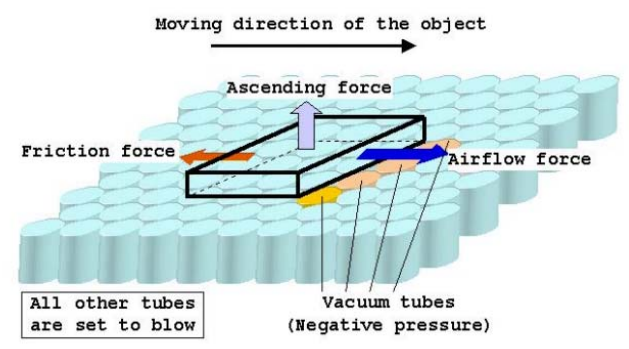

Figure 6. Mechanism of the active surface device

As shown in Fig. 6, two types of pressure are used in this system: positive (blowing) pressure and negative (vacuum) pressure. The blowing pressure creates an ascending force to lift the objects above the surface so that the friction between object and surface is decreased. The vacuum pressure creates a descending force, which draws the object to the surface. When ascending force is equal to the descending force, there is a balance and objects can be maintained there. On the other hand, a vacuum tube next to one blowing tube forms airflow together. This produces a lateral force of traction directed from the high pressure area to the low pressure area. Undoubtedly the objects will move with the airflow when the force of traction is larger than the friction force.

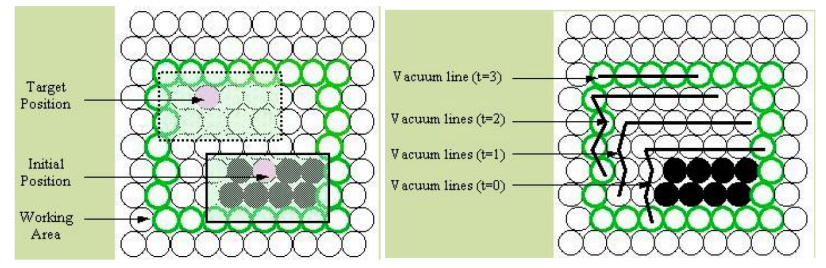

Figure 7. Mechanism of the active surface device Reservation of the working area and Successive vacuum lines for translation

The precise movement of the objects is the advantage of this system, which is realized in three steps. Firstly, the target position is selected artificially; it is the place where the center of the object should be placed. Then a rectangular working area is defined automatically to reserve all the tubes between the initial position and the target position (Fig. 7). Secondly, two lines of vacuum in vertical and horizontal directions are placed near the object; all the tubes not belonging to the current two lines are set to blow. Then airflow is formed to draw the object toward the desired position. Thirdly, the two lines of vacuum are moved step by step towards the target position until they reach the edges of working area [32].

In the Smart Surface project, another device for manipulating objects was also designed; it has similar structure of Ku's device, but the nozzles can only blow, not vacuum, so it is simpler. The product is carried on a thin air cushion and transported along the system by induced airflows. This induced airflow is an indirect effect of strong vertical air jets that pull the surrounding fluid.

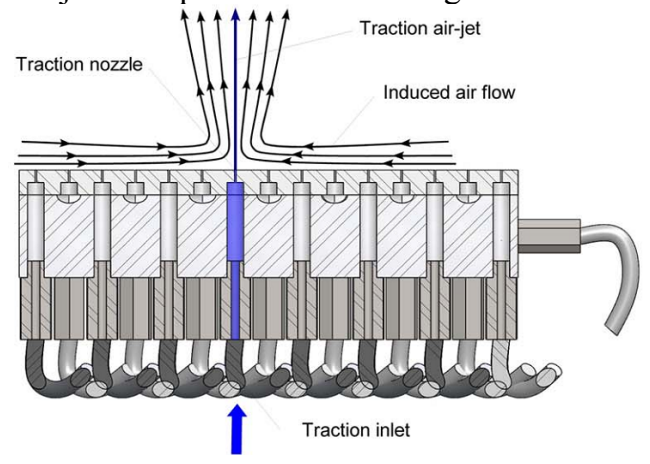

Figure 8. Global view that shows the generation of the induced air flow (the path of the air flow is darkened).

As shown in Fig. 8, the surface is consists of 225 holes in total; each one is $0.4 \mathrm{~mm}$ in diameter. The holes are divided into to two categories, 113 fixed holes and 112 specific holes, each specific hole connect one nozzle out of two to independent air inlets. The airflow spreads over these fixed holes and creates the air cushion under the object; the object is maintained in constant levitation due to the air cushion. The novelty is that the object can be moved on the table by generating strong vertical air jets through the specific nozzles of the surface. Each nozzle is driven by an independent solenoid valve. When a valve is open, a vertical air jet is generated. The air jet creates an induced air flow in the surrounding fluid that pulls the object toward the nozzle [33].

Researchers have done a lot of works based on this device, in order to identify the location of object and control it; A H $\infty$ robust controller is designed and implemented on the device (see [34]). In order to control the object precisely, distributed control architecture is presented (see [35]).

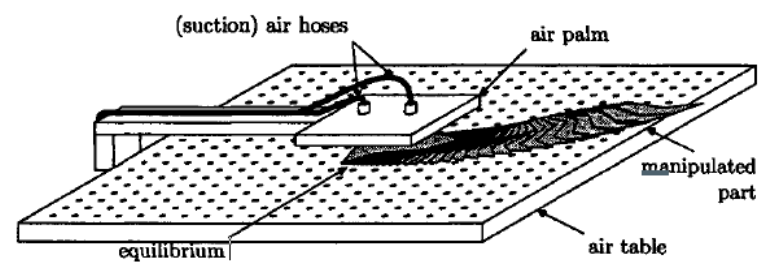

Figure 9. An air manipulator palm hovers above a standard air table to position, orient, lift, and cany away the object

The two projects presented before require a mechanically complex array of actuators, each of which must be individually controllable. Compared to this, Luntz and Moon [36-39] have introduced a simple method of generating distributed manipulation fields in which passive air flow fields is generated at only a small number of discrete points. It applies forces over the entire surface of object floating in the field. A properly established field will manipulate the object to a predictable equilibrium position and orientation. 
This system levitates objects by air cushion generated from a standard air table, and moves objects by generating the manipulation flow field on the top surface of the object (Fig. 9). The motion of objects is also accomplished in three steps: firstly, the "air palm", placed over the object, attracts it to its center and orients it using a pre-designed air field generated by flow sinks. Secondly, after a set time, the object is assumed to be oriented, suction increases, lifting the object and holding it firmly against the palm. Thirdly, the palm carries the object away with known orientation [40].

\section{Moving objects by other non-flow integrated approaches}

At present, there are also some conveying systems combined with the advantages of different levitation techniques. Air cushion is used for levitation and other techniques for moving.

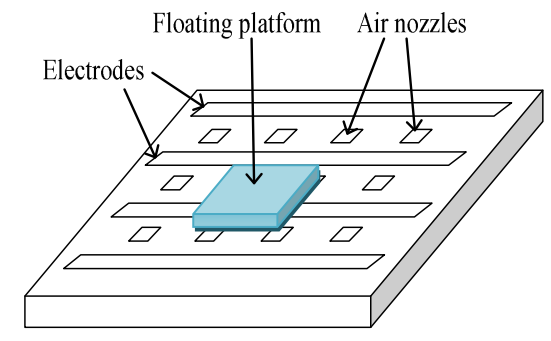

Figure 10. Illustration of a platform floating above the air nozzles and electrodes on the surface of the motor.

Pister has demonstrated a stable micro fabricated air bearing capable of levitating objects between $2 \mu \mathrm{m}$ and $1 \mathrm{~mm}$ thick with extremely low kinetic friction, and no static friction. In order to minimize friction, the moving elements of their design ride on a cushion of air supplied through nozzles in the fixed surface of the motor. These moving parts, or platforms, are then actuated by electrostatic fields generated by conductors in the surface of the motor [41] (Fig. 10).

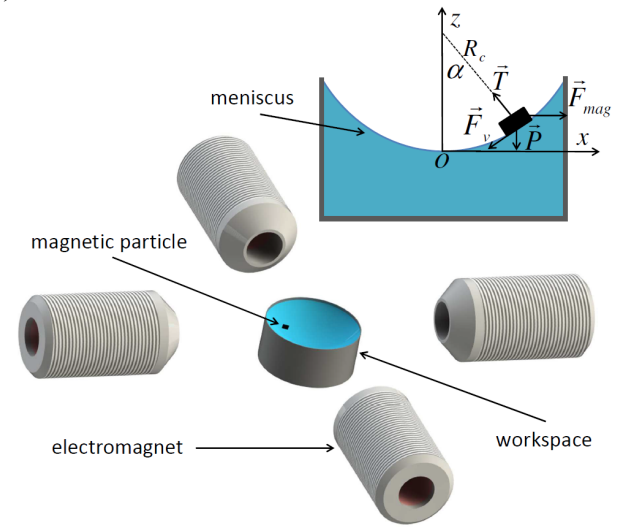

Figure 11. Illustration of of the planar magnetic device.

Dkhil proposes a new approach where objects are manipulated through magnetic fields. The modeling proposed in his paper is illustrated on a magnetic device composed of four electromagnets. The magnetic force produced induces the motion of the micro object on the air interface (see Fig. 11) [42]. Experimental measurements demonstrate the repeatability of the motion for objects larger than $50 \mu \mathrm{m}$ (the variation of the permanent position of an object of $100 \times 90 \times 25 \mu^{3}$ for a constant meniscus represents about $1 \%$ of its size). But, this device is designed without possible scalability properties.

\section{CONCLUSIONS}

This paper starts with the acknowledgment of the importance of levitation techniques and scalability and reconfigurability properties in modern conveyors. Four different technologies based on non-contact levitation are presented and compared. The air levitation has several advantages, such as clean, magnetic free and generating little heat, simple in design, easy to use and maintenance, and has less demanding on the environment.

A detailed analysis of many kinds of air levitation devices is made. According to the difference of moving mechanisms; they are summarized into three categories, i.e. inclined air jet, aerodynamic-traction and other non-flow integrated approaches. This provides a reference for selecting a reliable movement mechanism for future smart conveyors. Possible scalability of the different technique considered is presented.

In future work, we plan to design scalable smart conveyors based on smart blocks and air levitation. This work will be a sequel of the Smart Block project. We shall study in particular motion mechanisms of blocks and control laws of the distributed system.

\section{REFERENCES}

[1] N. Chaillet and S. Régnier, eds. Microrobotics for micromanipulation. John Wiley \& Sons, 2013.

[2] A. Berlin, D. Biegelsen, and P. Cheung. "Motion control of planar objects using large-area arrays of mems-like distributed manipulators", Micromechatronics, 2000.

[3] V. Vandaele, P. Lambert, and A. Delchambre, "Non-contact handling in microassembly: Acoustical levitation", Precision Engineering, vol. 4, 2005, pp.491-505.

[4] B. J. Kang, L. S. Hung, S. K. Kuo, S. C. Lin, and C. M . Liaw, "Hळ 2DOF control for the motion of a magnetic suspension positioning stage driven by inverter-fed linear motor", Mechatronics, vol. 7, 2003, pp.677-696.

[5] M. F. Zaeh, J. Zimmermann, A. Zitzmann, and M. Schilp, "Noncontact handling in microfabrication", Proceedings of the Euspen International Topical Conference, Aachen, Germany, 2003, pp.325328.

[6] F. Erzincanli, J. M. Sharp, and S. Erhal, "Design and operational considerations of a non-contact robotic system for non-rigid materials", Int J Mach Tools Manuf, vol. 4, 1998, pp.353-361.

[7] K. H. Park, S. K. Lee, J. H. Yi, S. H. Kim, Y. K. Kwak, and I. A. Wang, "Contactless magneticaly levitated siliconwafer transport system", Mechatronics, vol. 5, 1996, pp.591-610.

[8] J. R. Gao, C. D. Cao, and B. Wei, "Containerless processing of materials by acoustic levitation", Adv Space Res, vol. 10, 1999, pp.1293-1297.

[9] P. R. Southworth and G. R. Baxter, "Semiconductor wafer transport system”, US Patent 4540326, 1985.

[10] D. Belna, "Linear induction semiconductor wafer transportation apparatus", US Patent 4624617, 1986. 
[11] K. H. Park, K. Y. Ahn, S. H. Kim, Y. K. Kwak, and I. A. Wang, "Contactless magnetically levitated silicon wafer transport system", Mechatronics, 1996, vol.5, pp.591-610.

[12] K. H. Park, K. Y. Ahn, S. H. Kim, and Y. K. Kwak, "Wafer Distribution System for a Clean Room Using a Novel Magnetic Suspension Technique", IEEE/ASME Transactions on Mechatronics, 1998, vol. 1, pp. 73-77.

[13] P. Fowlai, H. Toshiro, Y. Koji and O. Koichi, "Non-contact Transportation System for Thin Glass Plate Utilizing combination of air bearing and electrostatic force", Proceedings of the $38^{\text {th }}$ SICE Annual Conference, 1999, pp.1053-1058.

[14] U. J. Jong and H. Toshiro, "Electrostatic Suspension of Dielectrics", IEEE Transactions on industrial electronics, 1998, vol.6, pp.938-946.

[15] J. U. Jeon, K. Y. Park, T. Higuchi, "Contactless suspension and transportation of glass panels by electrostatic forces", Sensors and Actuators A, 2007,vol. 2, pp.565-574.

[16] Y. Hashimoto,Y. Koike, and S. Ueha, "Transporting objects without contact using flexural traveling waves", Acoustical Society of America, 1998, vol.6, pp.3230-3233.

[17] W. Xie, "Theoretical and Experimental Study on the Characteristics of Pneumatic Levitation Using Porous Media", Northwestern Polytechnical University, 2002.

[18] Y. Ochiai, T. Hoshi, and J. Rekimoto, "Three-dimensional mid-air acoustic manipulation by ultrasonic phased arrays", PloS One, 2014, vol. 5, pp. e97590.

[19] X. Li, K. Kawashima, and T. Kagawa, "Analysis of vortex levitation", Experimental Thermal and Fluid Science, 2008, vol. 32, pp.1448-' 1454.

[20] S. Davis, J. O. Gray, and G. C. Darwin, "An end effector based on the bernoulli principle for handling sliced fruit and vegetables", Robotics and Computer-Integrated Manufacturing, 2008, vol. 24, pp.249-257.

[21] L. Guillaume, A. Delettre, and N. Le Fort-Piat. "A new aerodynamictraction principle for handling products on an air cushion." Robotics, IEEE Transactions on Vol. 27, $\mathrm{N}^{\circ}$ 2, 2011, pp.379-384.

[22] T. Hirata, T. Akashi, A. Bertholds, H.P. Gruber, A. Schmid, M.A. Gretillat, O.T. Guenat, N.F. de Rooij, A novel pneumatic actuator system realised by microelectro-discharge machining, in: Proceedings of IEEE Workshop on Micro Electro Mechanical Systems "MEMS '98', Heidelberg, Germany, 1998, pp.160-165.

[23] K. Salonitis, A. Stournaras, G. Tsoukantas, P. Stavropouls, and G. Chryssolouris, "Atheoretical and experimental investigation on limitations of pulsed laser drilling", Journal of Materials Processing Technology, 2007, vol. 1, pp.96-103.

[24] J. H. Kim, J. H. Boo, and Y. J. Kim, "Effect of stage control parameters on the FIB milling process", Journal of Materials Processing Technology, 2007, vol. 1, pp.96-103.

[25] R. Zeggari, R. Yahiaoui, J. Malapert, and J. F. Manceau, "Design and fabrication of a new two-dimensional pneumatic micro-conveyor", Sensors and Actuators A: Physical, 2010, vol. 1, pp.125-130.

[26] Y. Fukuta, Y. A. Chapuis, Y. Mita, and H. Fujita, "Design, fabrication, and control of MEMS-based actuator arrays for air-flow distributed micromanipulation", Microelectromechanical Systems, Journal of, 2006, vol. 4, pp.912-926.

[27] Y. Mita, M. Arai, A. Tixier, and H. Fujita, "Bulk micromachined durable air-flow microactuator array for robust conveyance systems," in Proc. 2001 Int. Conf. Solid-State Sensors and Actuators (Transducers), 2001, pp.710-713.

[28] D. El Baz, V. Boyer, J. Bourgeois, E. Dedu, and K. Boutoustous, "Distributed part differentiation in a smart surface", Mechatronics, 2012, vol. 5, pp.522-530.

[29] S. Mobes, G. J. Laurent, C. Clévy, L. Fort, B. Piranda, and J. Bourgeois, "Toward a 2D modular and self-reconfigurable robot for conveying microparts", In Design, Control and Software Implementation for Distributed MEMS, Second Workshop on IEEE, 2012, pp.7-13.
[30] D. El Baz, B. Piranda, and J. Bourgeois, “A Distributed Algorithm for a Reconfigurable Modular Surface”, IEEE International Parallel \& Distributed Processing Symposium and Workshops (IPDPSW), 2014, pp.1591-1598.

[31] S. Konishi and H. Fujita, "A conveyance system using air flow based on the concept of distributed micro motion systems", Microelectromechanical Systems, Journal of, 1994, vol. 2, pp.54-58.

[32] P. J. Ku, K. H. Winther, H. E. Stephanou H E, "Distributed control system for an active surface device", Robotics and Automation, Proceedings 2001 ICRA. IEEE International Conference on, IEEE, 2001, vol. 4, pp.3417-3422.

[33] G. J. Laurent, A. Delettre, and N. Le Fort-Piat, “A new aerodynamictraction principle for handling products on an air cushion", Robotics, IEEE Transactions on, 2011, vol.2, pp.379-384.

[34] A. Delettre, G. J. Laurent, Y.Hadda, and L. Fort-Piat, "Robust control of a planar manipulator for flexible and contactless handling", Mechatronics, 2012, vol. 6, pp.852-861.

[35] K. Boutoustous, G. Laurent, E. Dedu, L. Matignon, J. Bourgeois, and N. Le Fort-Piat, "Distributed control architecture for smart surfaces", 2010 IEEE/RSJ International Conference on Intelligent Robots and Systems (IROS), 2010, pp.2018-2024.

[36] J. Luntz and H. Moon, "Distributed manipulation with passive air flow", In Proc. of the IEEE Int. Conf. on Intelligent Robots and Systems, 2001, pp.195-201.

[37] H. Moon and J. Luntz, "Distributed manipulation of flat objects with two airflow sinks", IEEE Transactions on Robotics, 2006, vol.6, pp.1189-1201.

[38] K. Varsos and J. Luntz, "Superposition methods for distributed manipulation using quadratic potential force fields", IEEE Transactions on Robotics, 2006, vol. 6, pp.1202-1215.

[39] K. Varsos, H. Moon, and J. Luntz, "Generation of quadratic potential force fields from flow fields for distributed manipulation”, IEEE Transactions on Robotics, 2006, vol.1, pp.108-118.

[40] J. Luntz, H. Moon, "Distributed manipulation with passive air flow", IEEE/RSJ International Conference on Intelligent Robots and Systems, 2001, vol. 1, pp.195-201.

[41] K. S. J. Pister, R. Fearing, R. Howe, "A planar air levitated electrostatic actuator system", Micro Electro MechanicalSystems, Proceedings, An Investigation of Micro Structures, Sensors, Actuators, Machines and Robots, IEEE, 1990, pp.67-71.

[42] M. Dkhil, A. Bolopion, S. Regnier, and M. Gauthier, "Modeling and experiments of high speed magnetic micromanipulation at the air/liquid interface", 2014 IEEE/RSJ International Conference on Intelligent Robots and Systems (IROS 2014), 2014, pp.4649-4655. 